\title{
ON THE PROBABILITY OF ADAPTATION ERROR IN MIMO SYSTEMS
}

\author{
Ping-Heng Kuo Peter J. Smith \\ Department of Electrical and Computer Engineering \\ University of Canterbury \\ Christchurch, New Zealand \\ Email: phk15@student.canterbury.ac.nz
}

\begin{abstract}
In this paper, we are interested in evaluating the probability of adaptation error (PAE) in MIMO systems using adaptive modulation or transmission rate. We attack the problem from two distinct perspectives. Firstly, for a certain rate-feedback system, the PAE is computed by building a Markov model for the channel capacity. The transition probabilities between rate states during the feedback period are approximated using a novel analytical result for the level crossing rate (LCR) of MIMO capacity. Secondly, the impact of channel estimation error on adaptive modulation over eigenmodes is considered. By utilizing the joint dynamic statistics of the eigenvalues, the instantaneous probabilities of choosing inappropriate modulation schemes can be calculated.
\end{abstract}

\section{INTRODUCTION}

Many adaptive MIMO systems have been suggested to increase throughput, and it is important to consider the effects of the mobile channel environment on system performance. In this paper, the probability of adaptation error (PAE) is considered, which provides some important insights into dynamic bitbudget preparation. In particular, we focus on evaluating the probability of inappropriate rate or modulation selection due to channel time variation or channel estimation error. As part of the probability calculations a novel analytical result for the level crossing rate (LCR) for MIMO channel capacity is also derived. The main contributions of the paper are elaborated below.

Firstly, we examine the rate-feedback scheme in [1]. Since capacity represents the maximum rate that the channel can support, the rate-feedback scheme computes the present channel capacity at the receiver, and sends this information to the transmitter via a feedback link. For such a system, PAE is the probability of rate-assignment error (PORAE). For efficient feedback purposes [1], the capacity is partitioned into several discrete quantities as a finite list of states, each corresponding to a specific transmission rate. Thus, to evaluate how channel fluctuations affect PAE, a finite-state Markov chain (FSMC) can be used. A FSMC is a popular model for the wireless channel due to its simplicity $[2,3]$. Only a handful of papers have modeled MIMO channels by a FSMC [4]. Recently, we have became aware that FSMC modeling for MIMO capacity has also appeared in [5] with a similar motivation. However, the method used to approximate the transition probabilities is different.

Secondly, our scope moves to another scenario. It is wellknown that different modulation schemes can be applied to the multiple eigenmodes in MIMO channels to increase the trans- mission speed $[6,7]$. Here, we concentrate on the instantaneous PAE, which is the probability of modulation-assignment error (POMAE) over the multiple eigenmodes, caused by channel estimation error. The joint dynamic statistics of the eigenvalues are exploited, and the results are verified through simulations.

The organization of the paper is as below. Section II provides some fundamental background and mathematical formulations for MIMO channels. We construct a FSMC for channel capacity in section III. The LCR for MIMO channel capacity is also derived in this section in order to calculate the transition probabilities. In section IV, the adaptive modulation scheme is evaluated using the joint dynamic statistics of the eigenvalues. Finally, conclusions are drawn in section V.

\section{BACKGROUND}

For a MIMO system with $N_{T}$ antennas at the transmitter and $N_{R}$ antennas at the receiver, the channel matrix, $\boldsymbol{H}$, is an $N_{R} \times$ $N_{T}$ matrix. Assuming a flat Rayleigh fading channel, elements of $\boldsymbol{H}$ are i.i.d. complex Gaussian variables denoted $\mathrm{eN}(0,1)$. Also we define $m=\min \left(N_{R}, N_{T}\right)$ and $n=\max \left(N_{R}, N_{T}\right)$.

By applying the singular value decomposition (SVD), the channel matrix $\boldsymbol{H}$ can be written as

$$
\boldsymbol{H}=\boldsymbol{U} D \boldsymbol{V}^{*},
$$

where $\boldsymbol{U} \in \mathcal{C}^{N_{R} \times N_{R}}$ and $\boldsymbol{V} \in \mathcal{C}^{N_{T} \times N_{T}}$ are unitary matrices. $D$ is a diagonal matrix whose nonzero elements $\sqrt{\lambda_{1}} \geq \sqrt{\lambda_{2}} \geq$ $\ldots \geq \sqrt{\lambda_{m}}$ are ordered singular values of the channel matrix $\boldsymbol{H}$. With this notation, assuming equal power allocation, the overall MIMO channel capacity can be written as [8]:

$$
C(t)=\sum_{i=1}^{m} \log _{2}\left(1+\frac{P}{N_{T}} \lambda_{i}(t)\right) \quad \text { bits } / s / H z
$$

where $P$ is the signal-to-noise ratio (SNR).

\section{PORAE FOR RATE-FEEDBACK SYSTEMS: MARKOV MODEL FOR CAPACITY PROCESS}

A rate-feedback scheme has been discussed in [1] where the capacity is quantized into several discrete values that are known at both transmitter and receiver. These quantized values can represent states and we model the capacity over time as a FSMC over these states. We assume that the receiver possesses perfect knowledge of the channel, so the selection of rate is made at the receiver and the transmitter is informed via a feedback link. Hence, the time-varying nature of the mobile channel may cause the selected rate to become outdated when applied at the transmitter. Our goal here is to find the probability that the current rate selection becomes inappropriate (either too high or too low) during the feedback time period. 
For the sake of simplicity, we assume that the channel timevariation is governed by the Jakes model with autocorrelation function $J_{0}\left(2 \pi f_{D} \tau\right)$ [9], where $f_{D}$ and $\tau$ are the Doppler frequency and time displacement respectively ( $\tau$ is the feedback period in this case). In addition, the channel variation is assumed to be slow enough so that only transitions between adjacent states are possible.

\section{A. State Partition and Transition Probabilities}

To partition the capacity process into finite states, we employ the quantization method proposed in [1] for feedback purposes. If $b$ is any integer, a list of $2 b+1$ possible rates, $\mathcal{L}_{b}$, can be generated as:

$$
\begin{aligned}
\mathcal{L}_{b}= & \{0, \mu(1-b \alpha), \ldots, \mu(1-\alpha), \mu, \\
& \mu(1+\alpha), \ldots, \mu(1+(b-1) \alpha)\},
\end{aligned}
$$

where $\mu$ is the mean rate, and $\alpha$ is an arbitrary proportion of the mean rate, known as granularity. Thus, the rate $\mu(1+i \alpha)$ is selected whenever the capacity lies between $\mu(1+i \alpha)$ and $\mu(1+(i+1) \alpha)$. Hence, the states can be denoted $S_{1}, S_{2}, \ldots S_{2 b+1}$ where $S_{1}$ occurs when $C<\mu(1-b \alpha), S_{j}$ occurs when $\mu(1-(b-j+2) \alpha) \leq C<\mu(1+(b-j+1) \alpha)$ for $2 \leq j \leq 2 b$ and $S_{2 b+1}$ occurs when $\mu(1+(b-1) \alpha) \leq C$. In a slight divergence from [1], we have an extra state, "0", to indicate channel outage, when the channel is too weak to support transmission. To construct a FSMC, we now need to determine the transition probabilities between any state and its neighbor states. Both [2] and [3] have modeled the Rayleigh fading channel as a FSMC, by using the LCR method to approximate transition probabilities. Define the transition probabilities from state $S_{k}$ to $S_{k+1}$ by $\operatorname{Prob}(k, k+1)$ and from state $S_{k}$ to $S_{k-1}$ by $\operatorname{Prob}(k, k-1)$, then we have:

$$
\begin{gathered}
\operatorname{Prob}(k, k+1) \approx \frac{\operatorname{LCR}\left(T_{k+1}\right) \tau}{\operatorname{Prob}\left(C \in S_{k}\right)}, k=1,2, \ldots, 2 b \\
\operatorname{Prob}(k, k-1) \approx \frac{\operatorname{LCR}\left(T_{k}\right) \tau}{\operatorname{Prob}\left(C \in S_{k}\right)}, k=2,3, \ldots, 2 b+1 .
\end{gathered}
$$

Note that $T_{k+1}$ represents the boundary between $S_{k}$ and $S_{k+1}$. Since previous work suggests that MIMO capacity can be approximated by a Gaussian variable $[10,1], \operatorname{Prob}\left(C \in S_{k}\right)$ can be evaluated easily using the mean and variance, which can be computed from the results in [8] and [10] respectively. The transition probabilities give the probabilities of incorrect rate selection in the following way. $\operatorname{Prob}(k, k \pm 1)$ is the probability that state $k \pm 1$ is correct after the feedback but the channel was estimated as supporting rate $k$ before feedback. Hence state $k$ is incorrectly used. As a consequence, the probability of incorrectly using state $i$, given that state $i$ is used, is defined by $\operatorname{PORAE}_{i}=\operatorname{Prob}(i, i+1)+\operatorname{Prob}(i, i-1)$. The absolute probability of incorrectly using state $i$ is $\operatorname{Prob}\left(C \in S_{i}\right) \times \mathrm{PORAE}_{i}$.

\section{B. LCR for MIMO Capacity}

To the best of our knowledge, preceding studies on capacity LCR have required simulation to compute either the rates themselves or the autocorrelation functions required for analytical approximation [11, 12]. In this paper we remove the need for simulation and derive an analytical formula to approximate the LCR as elaborated below.

MIMO capacity is approximated by a Gaussian process and the corresponding LCR formula is available in [9]:

$$
\operatorname{LCR}_{\text {Gaussian }}(T)=\frac{\sqrt{-\ddot{R}(0)}}{2 \pi} \exp \left[\frac{-1}{2}\left(\frac{T-\mu}{\sigma}\right)^{2}\right]
$$

where $\mu$ and $\sigma$ are the mean and standard deviation of the capacity respectively. The double derivative, $\ddot{R}(0)$, is the curvature of the standardized autocorrelation function at $\tau=0$. From [8] and [10] the mean and variance are available and so only $\ddot{R}(0)$ is required. If $C(t)$ is the capacity at time $t$ then $\ddot{R}(0)$ is given by $2 \Gamma / \sigma^{2}$ where $\Gamma$ is the coefficient of $\tau^{2}$ in $E[C(t) C(t+\tau)]$. Using (2), $C(t)$ and $C(t+\tau)$ can be written in terms of $\lambda_{i}(t)$ and $\lambda_{i}(t+\tau)$. Furthermore, if we define $\triangle \lambda_{i}=\lambda_{i}(t+\tau)-\lambda_{i}(t)$, the stochastic differential equation (SDE) in [13] can be modified to acquire:

$$
\triangle \lambda_{i} \approx 2 \pi f_{D}\left[\sqrt{\lambda_{i}(t)} Z_{i} \tau+\pi f_{D}\left(n+\Phi_{i}-\lambda_{i}(t)\right) \tau^{2}\right]
$$

where

$$
\Phi_{i}=\sum_{k \neq i} \frac{\lambda_{i}(t)+\lambda_{k}(t)}{\lambda_{i}(t)-\lambda_{k}(t)}
$$

and $Z_{i}$ is an independent $\mathcal{N}(0,1)$ variable. Thus, following [14], we can substitute for $\lambda_{i}(t)$ and $\lambda_{i}(t+\tau)$ in (2) and evaluate $E[C(t) C(t+\tau)]$ for small values of $\tau$. Finding the coefficient of $\tau^{2}$ in the resulting expression gives, after a little algebra,

$$
\ddot{R}(0)=\frac{5.77 P \pi^{2} f_{D}^{2}}{\sigma^{2}} \sum_{i=1}^{m} \sum_{k=1}^{m} \mathrm{E}\left(A_{i}\right)
$$

where $\mathrm{E}\left(A_{i}\right)$ is the expectation of

$$
\log _{2}\left(1+\frac{P}{N_{T}}\right)\left[\frac{n+\Phi_{i}-\lambda_{i}(t)}{N_{T}+P \lambda_{i}(t)}-\frac{P \lambda_{i}(t)}{\left[N_{T}+P \lambda_{i}(t)\right]^{2}}\right]
$$

Hence $\ddot{R}(0)$ can be calculated and used in (6) to evaluate LCR. A more detailed derivation of the capacity LCR will appear in [15]. Note that computation of $E\left(A_{i}\right)$ can be performed exactly due to the simple form of the joint eigenvalue density given in [8]. Nevertheless, it is awkward and an algebraic manipulation package is helpful. Figure. 1 shows the LCR comparison between simulated results and (6) for a $(2,2)$ MIMO channel. Results not shown here demonstrate that simulated LCR curves are equally well approximated by this method for all systems up to $(4,4)$. Results for larger systems may be even better since the Gaussian approximation will further improve.

\section{Numerical Examples}

By using the technique described above, results have been obtained for the following scenario. Consider a $(2,4)$ MIMO system with a SNR of $P=9 d B$. Assuming a carrier frequency of $5.725 \mathrm{GHz}$, with a user speed of $5 \mathrm{~km} / \mathrm{hr}$ and a feedback delay, $\tau$, of $1 \mathrm{~ms}$, we have $f_{D} \tau=0.0265$. For this scenario, 


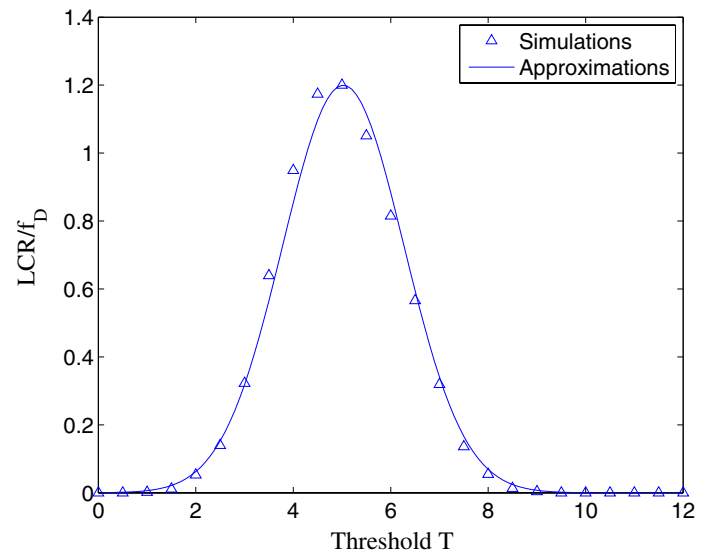

Figure 1: LCR for the capacity of a $(2,2)$ MIMO channel.

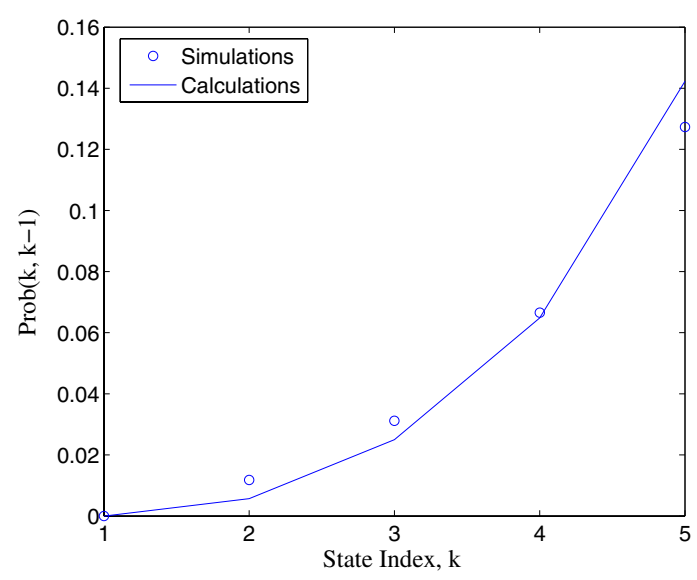

Figure 2: A comparison between simulated and calculated transition probabilities.

$\mu$ is approximately 7.5 , and the range of capacity values can be conveniently partitioned into 5 states by choosing $b=2$ and $\alpha=1.5$. All results agree with the simulations quite well and some comparisons are shown in Fig. 2 and Fig.3. The full transition matrix for the FSMC, computed using (4) and (5) is shown in Table 1.

As we can see in Fig. 3, it is most likely for the system to choose the wrong rate in "state 1". Although the approximation deteriorates at the extreme states, the FSMC illustrates the essential temporal behavior of MIMO channel capacity with an acceptable accuracy for such a simple model. The size of the error probabilities are interesting with values ranging from $8 \%$ to $26 \%$. Clearly, the moderate feedback delay can have considerable impact on rate selection.

\section{InStANTANEOUS POMAE ON EIGENMODES With IMPERFECT CHANNEL ESTIMATION}

It has been suggested that adaptive modulation can be applied to the channel eigenmodes to significantly improve the throughput of MIMO systems [6, 7]. In this section, we inves-

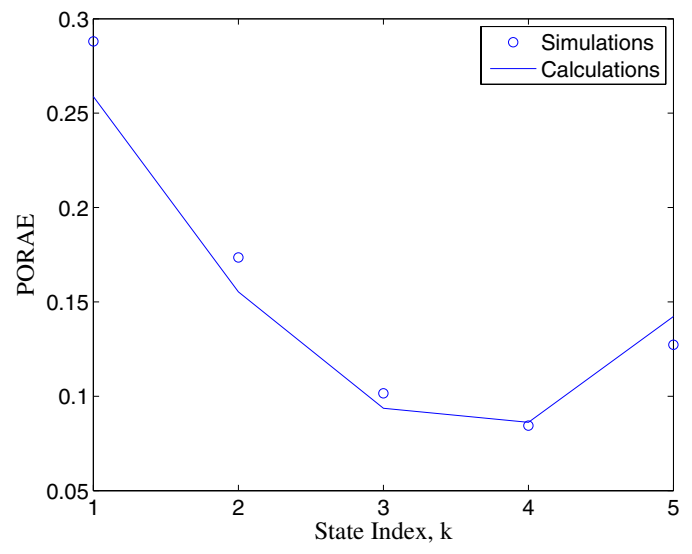

Figure 3: A comparison between simulated and calculated PORAE for a $(2,4)$ rate-feedback system.

Table 1: Transition matrix and PORAE results for the capacity of a $(2,4)$ MIMO channel $(\mathrm{k}=$ state index $)$.

\begin{tabular}{lllll}
$\mathrm{k}$ & $\operatorname{Prob}(\mathrm{k}, \mathrm{k}-1)$ & $\operatorname{Prob}(\mathrm{k}, \mathrm{k})$ & $\operatorname{Prob}(\mathrm{k}, \mathrm{k}+1)$ & PORAE \\
\hline \hline 1 & - & 0.7412 & 0.2588 & 0.2588 \\
2 & 0.0057 & 0.8447 & 0.1496 & 0.1553 \\
3 & 0.0250 & 0.9064 & 0.0686 & 0.0936 \\
4 & 0.0648 & 0.9138 & 0.0214 & 0.0862 \\
5 & 0.1423 & 0.8577 & - & 0.1423 \\
\hline
\end{tabular}

tigate the instantaneous probability that channel estimation errors will mislead the system into choosing inappropriate modulation types. Such a probability is referred to as the instantaneous POMAE and is conditional on the current channel matrix.

\section{A. Channel Estimation Error Model}

We consider a simple channel estimation error model, where the estimated channel $\widehat{\boldsymbol{H}}$ is written as [16]:

$$
\widehat{\boldsymbol{H}}=\boldsymbol{H}+\triangle \boldsymbol{H} .
$$

In (10), $\boldsymbol{H}$ is the true channel, as in Section II., and $\triangle \boldsymbol{H}$ is an additive error matrix with i.i.d complex Gaussian elements, $\operatorname{eN}\left(0, \sigma_{e}^{2}\right)$. Assuming a maximum-likelihood estimator is used with $L$ training symbols, then we have [16]:

$$
\sigma_{e}^{2}=\frac{N_{T}}{P L}
$$

where $P$ and $N_{T}$ are defined as before.

\section{B. Joint Transition Density of Eigenvalues}

It has been shown that the $m$ eigenvalues of an i.i.d complex Brownian correlation matrix, are equivalent to $m$ independent squared Bessel processes conditioned never to collide [13]. Define one such eigenvalue at time $t=0$ as $w$ and the same eigenvalue at time $t>0$ is denoted $\widehat{w}$. The eigenvalue has 
the transition density [13]:

$$
p(\widehat{w} \mid w)=\frac{1}{2 t}\left(\frac{\widehat{w}}{w}\right)^{\frac{v}{2}} \exp \left[\frac{-w-\widehat{w}}{2 t}\right] I_{v}\left(\frac{\sqrt{w \widehat{w}}}{t}\right)
$$

where $v=n-m$ and $I_{v}$ is the $v^{t h}$ order modified Bessel function. Note that equation (12) gives the conditional density of the eigenvalue $\widehat{w}$ at time $t$ conditioned on the value $w$ at time zero. The complex matrix process considered in (12), has entries which are Brownian motion processes, defined by $\boldsymbol{B}_{t}=$ $\boldsymbol{B}_{t=0}+\mathcal{C} \mathcal{N}(0,2 t)$. This process has the same structure as the estimation error model in (10). We can therefore modify (12) by using $2 t=\sigma_{e}^{2}$, which gives:

$$
p(\widehat{\lambda} \mid \lambda)=\frac{P L}{N_{T}}\left(\frac{\widehat{\lambda}}{\lambda}\right)^{\frac{v}{2}} \exp \left[\frac{-P L(\lambda+\widehat{\lambda})}{N_{T}}\right] I_{v}\left(\frac{2 P L \sqrt{\lambda \widehat{\lambda}}}{N_{T}}\right)
$$

In (13), $\lambda$ and $\widehat{\lambda}$ are eigenvalues of $\boldsymbol{H}$ and $\widehat{\boldsymbol{H}}$ respectively. Since these eigenvalues are conditioned never to collide, the ordering $\lambda_{1}>\lambda_{2}>\ldots>\lambda_{m}$ is preserved and the corresponding joint transition density of the eigenvalues is given as $[17,18]$ :

$$
p\left(\widehat{\lambda_{1}}, \ldots, \widehat{\lambda_{m}} \mid \lambda_{1}, \ldots, \lambda_{m}\right)=\frac{\prod_{i>j}\left(\widehat{\lambda_{j}}-\widehat{\lambda_{i}}\right)}{\prod_{i>j}\left(\lambda_{j}-\lambda_{i}\right)} \times G
$$

where

$$
G=\operatorname{det}\left[\begin{array}{cccc}
p\left(\widehat{\lambda_{1}} \mid \lambda_{1}\right) & p\left(\widehat{\lambda_{2}} \mid \lambda_{1}\right) & \cdots & p\left(\widehat{\lambda_{m}} \mid \lambda_{1}\right) \\
p\left(\widehat{\lambda_{1}} \mid \lambda_{2}\right) & p\left(\widehat{\lambda_{2}} \mid \lambda_{2}\right) & & \vdots \\
\vdots & & \ddots & \vdots \\
p\left(\widehat{\lambda_{1}} \mid \lambda_{m}\right) & \cdots & \cdots & p\left(\widehat{\lambda_{m}} \mid \lambda_{m}\right)
\end{array}\right]
$$

Thus, the instantaneous probabilities of incorrect modulation choice can be obtained by fixing $\lambda_{1}, \ldots, \lambda_{m}$ and integrating (14) with respect to $\widehat{\lambda_{1}}, \ldots, \widehat{\lambda_{m}}$ over the regions which cause an incorrect choice. This is clearly difficult for large systems but is manageable by numerical integration when $m=2$.

\section{Case Study}

As examples for simulation, $(2,2)$ and $(2,4)$ systems are considered. Assuming five options for modulation types: Outage, BPSK, QPSK, 8-PSK and 16-QAM, there are 10 possible sets of modulations over the two eigenmodes, as tabulated in Table 2. The switching criterion are SNR levels. The boundaries are the minimum required SNR levels, $\gamma$, for a certain modulation scheme to achieve the desired performance based on a target BER, $p_{e}$, set at $10^{-3}$. The SNR on the $i^{t h}$ eigenmode is $P \lambda_{i}$. To determine the $\gamma$ boundaries for switching modulations, the approximate method for M-PSK in [19] is used:

$$
\gamma_{M P S K} \approx-\frac{1}{8} \ln \left(4 p_{e}\right) 2^{1.94 \frac{\ln (M)}{\ln (2)}}
$$

Also, for square M-QAM [20]:

$$
\gamma_{M Q A M} \approx-\frac{2(M-1) \ln \left(5 p_{e}\right)}{3}
$$

Table 2: Possible combination sets of modulation schemes in the MIMO systems with two eigenmodes.

\begin{tabular}{lll} 
Set & $\lambda_{1}$ & $\lambda_{2}$ \\
\hline \hline 1 & BPSK & Outage \\
2 & QPSK & Outage \\
3 & QPSK & BPSK \\
4 & 8-PSK & Outage \\
5 & 8-PSK & BPSK \\
6 & 8-PSK & QPSK \\
7 & 16-QAM & Outage \\
8 & 16-QAM & BPSK \\
9 & 16-QAM & QPSK \\
10 & 16-QAM & 8-PSK \\
\hline
\end{tabular}

Table 3: The corresponding eigenmode gain regions for different modulations.

\begin{tabular}{ll} 
Modulations & Corresponding Eigenmode Gain Regions \\
\hline \hline BPSK & $0.3310 \leq \lambda<1.2702$ \\
QPSK & $1.2702 \leq \lambda<4.8738$ \\
8-PSK & $4.8738 \leq \lambda<6.6229$ \\
16-QAM & $6.6229 \leq \lambda$ \\
\hline
\end{tabular}

By assuming $P=9 d B$ on both eigenmodes, the corresponding eigenmode gains for these modulation types are summarized in Table 3. Furthermore, we assume $L=5$ training symbols are used which gives $\sigma_{e}^{2}=0.05$. In Fig. 4 and Fig. 5 we present two realizations of $\lambda_{1}, \lambda_{2}$ for a $(2,2)$ and $(2,4)$ system, respectively. The first realization has a small minimum eigenvalue and $\lambda_{1}=1.6678, \lambda_{2}=0.0234$. For these values the modulation scheme should be "set 2". In Fig. 4, however, it can be seen that there is a probability of about 0.1 that channel estimation error causes the incorrect selection of "set 1 ". This type of error reduces the achievable transmission speed but does not reduce the error performance. In the second realization, we have a larger minimum eigenvalue, such that $\lambda_{1}=6.0252$ and $\lambda_{2}=0.7537$. For these values "set 5" should be selected. As shown in Fig. 5, there is a probability of around 0.3 that the system will incorrectly select "set 8", which may lead to significant degradation of error performance. In both cases, we see excellent agreement between the calculated probabilities and the simulation results.

\section{CONCLUSIONS}

In this paper, we have considered the effects of channel variation and estimation on adaptive systems. The contributions are in two areas. Firstly, for rate-feedback systems, we have constructed a FSMC model for the MIMO channel capacity. An approximate LCR for MIMO capacity has been derived, in order to determine the transition probabilities from one rate-state to another during the feedback period. This allows an analytical evaluation of PORAE, and reasonably high levels of incorrect rate selection are observed for moderate feedback delays. The second part dealt with a different scenario where adaptive 


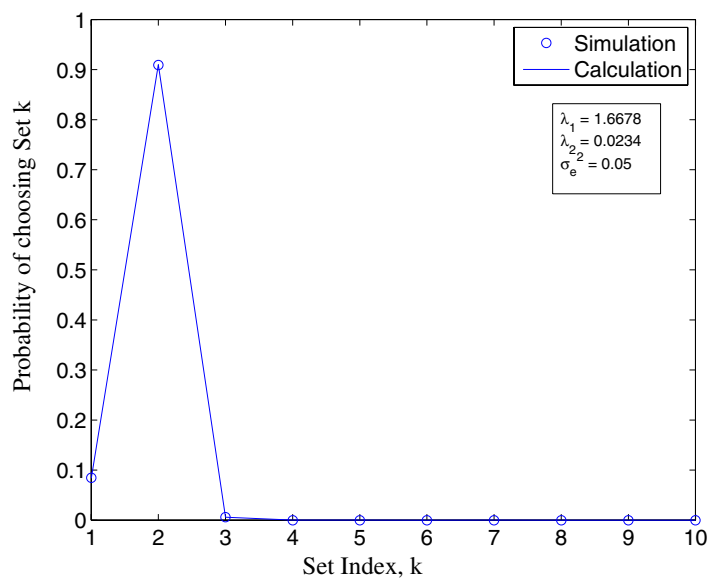

Figure 4: Comparison between simulated and calculated probabilities of choosing set $\mathrm{k}$ after channel estimation for a $(2,2)$ system.

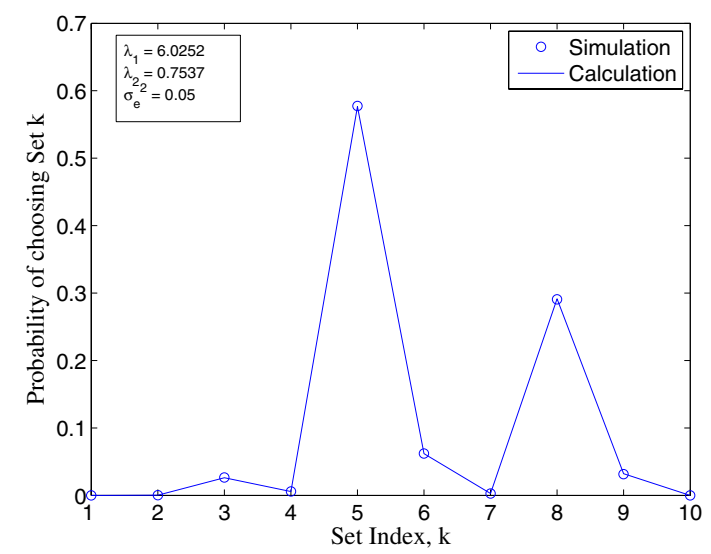

Figure 5: Comparison between simulated and calculated probabilities of choosing set $\mathrm{k}$ after channel estimation for a $(2,4)$ system.

modulation is applied to multiple eigenmodes. We presented a method to compute the probabilities of incorrect modulation selection (POMAE) due to channel estimation error. Results, using a physically motivated model for the channel estimation error, show that, in some cases, small errors can affect the system quite significantly.

\section{REFERENCES}

[1] B. M. Hochwald, T. L. Marzetta, and V. Tarokh, "Multiple-antenna channel hardening and its implications for rate feedback and scheduling," IEEE Trans. Inform. Theory, vol. 50, pp. 1893-1909, Sept. 2004.

[2] H. S. Wang and N. Moayeri, "Finite-state Markov channel - a useful model for radio communication channels," IEEE Trans. Veh. Technol., vol. 44, pp. 163-171, Feb. 1995.

[3] Q. Zhang and S. A. Kassam, "Finite-state Markov models for Rayleigh fading channels," IEEE Trans. Commun., vol. 47, pp. 1688-1692, Nov. 1999.

[4] R. D. Souza, J. Garcia-Frias, and A. M. Haimovich, "Using hidden Markov models to improve performance of space-time codes in MIMO flat fast-fading channels," in Proc. IEEE VTC Spring, 2004, pp. 13051309.

[5] S. Vaihunthan, S. Haykin, and M. Sellathurai, "MIMO channel capacity modeling using Markov models," in Proc. IEEE VTC Spring, 2005, pp. 126-130.

[6] J. C. Roh and B. D. Rao, "Adaptive modulation for multiple antenna channels," in Proc. Asilomar Conf. on Sig. Sys. and Comp., vol. 1, Nov. 2002, pp. 526-530.

[7] B. N. Getu, J. B. Andersen, and J. R. Farserotu, "MIMO systems: optimizing the use of eigenmodes," in Proc. IEEE PIMRC, 2003, pp. 1129 1133.

[8] I. E. Telatar, "Capacity of multi-antenna Gaussian channels," European Trans. on Telecomm. Related Technol., vol. 10, pp. 585-595, Nov. 1999.

[9] W. C. Jakes, Microwave Mobile Communications. Piscataway, NJ: IEEE Press Classic Reissue, 1995.

[10] P. J. Smith and M. Shafi, "An approximate capacity distribution for MIMO systems," IEEE Trans. Commun., vol. 52, pp. 887-890, June 2004.

[11] A. Giorgetti, M. Chiani, M. Shafi, and P. J. Smith, "Level crossing rates and MIMO capacity fades: Impacts of spatial/temporal channel correlation," in Proc. IEEE ICC, May 2003, pp. 3046-3050.

[12] B. O. Hogstad and M. Patzold, "Capacity studies of MIMO channel models based on the geometrical one-ring scattering model," in Proc. IEEE PIMRC, 2004, pp. 1613-1617.

[13] W. Konig and N. O'Connell, "Eigenvalues of the Laguerre process as non-colliding squared Bessel processes," Elect. Comm. in Prob., vol. 6, pp. 107-114, 2001.

[14] P. J. Smith, P.-H. Kuo, and L. M. Garth, "Level crossing rates for MIMO channel eigenvalues: implications for adaptive systems," in Proc. IEEE ICC, May 2005, pp. 2442-2446.

[15] P.-H. Kuo, P. J. Smith, and L. M. Garth, "Level crossing analysis of MIMO eigenmodes and associated channel metrics," in preparation.

[16] V. T. Ermolayev, A. G. Flaksman, I. P. Kovalyov, and I. M. Averin, "Weight error loss in MIMO systems with adaptive transmit and receive beamformers," in Proc. IEEE Int. Conf. Ant. Theo. and Tech., Sept. 2003, pp. 333-336.

[17] G. Ben-Arous and S. Peche, "Universality of local eigenvalue statistics for some sample covariance matrices," Commun. on Pure and Appl. Math., vol. 58, pp. 1316-1357, 2005.

[18] S. Lawi, "A characterization of markov processes enjoying the timeinversion property," 2005, submitted to Probability Theory and Related Fields.

[19] S. T. Chung and A. J. Goldsmith, "Degrees of freedom in adaptive modulation: a unified view," IEEE Trans. Commun., vol. 49, pp. 1561-1571, Sept. 2001.

[20] S. Zhou and G. B. Giannakis, "Adaptive modulation for multiantenna transmissions with channel mean feedback," IEEE Trans. Wireless Commun., vol. 3, pp. 1626-1636, Sept. 2004. 